\title{
Gilbert Damping in Single and Multilayer Ultrathin Films: Role of Interfaces in Nonlocal Spin Dynamics
}

\author{
R. Urban, G. Woltersdorf, and B. Heinrich \\ Simon Fraser University, 8888 University Drive, Burnaby, British Columbia, V5A 1S6, Canada
}

(Received 5 June 2001; published 2 November 2001)

\begin{abstract}
Unique features of the Gilbert damping in magnetic multilayers were investigated by ferromagnetic resonance (FMR) using magnetic single and double layer structures prepared by molecular beam epitaxy. The FMR linewidth for the Fe films in the double layer structures was larger than the FMR linewidth in the single Fe films having the same thickness. The additional FMR linewidth scaled inversely with the film thickness, and increased linearly with increasing microwave frequency. These results demonstrate that a transfer of electron angular momentum between the magnetic layers leads to additional relaxation torques.
\end{abstract}

DOI: 10.1103/PhysRevLett.87.217204

PACS numbers: 75.40.Gb, 75.70.-i, 76.50.+g, 76.60.Es

Introduction. - A new class of electronic devices whose operation is based upon the spin-polarized character of the electronic carriers in magnetic multilayers is being developed with an impressive rate of progress and determination. Magnetoelectronics and spintronics are terms used to mark the development of such devices. Spintronics is based on the unique properties of magnetic multilayers. It is paramount to its further development to find a better understanding of the spin dynamics and relaxation processes in the nanosecond time regime.

The spin dynamics in the classical limit can be described by the Gilbert equation of motion,

$$
\frac{1}{\gamma} \frac{\partial \boldsymbol{M}}{\partial t}=-\left[\boldsymbol{M} \times \boldsymbol{H}_{\mathrm{eff}}\right]+\frac{G}{\gamma^{2} M_{s}^{2}}\left[\boldsymbol{M} \times \frac{\partial \boldsymbol{M}}{\partial t}\right],
$$

where $\gamma$ is the absolute value of the electron spectroscopic splitting factor, and $M_{s}$ is the saturation magnetization. The first term on the right-hand side represents the precessional torque and the second term represents the wellknown Gilbert damping torque [1]. In the limit of small damping, $G /\left(\gamma M_{s}\right) \ll 1$, Gilbert relaxation torque and Landau-Lifshitz relaxation terms, $-\frac{G}{\gamma M_{s}^{2}}[\boldsymbol{M} \times \boldsymbol{M} \times$ $\left.\boldsymbol{H}_{\text {eff }}\right]$, are equivalent. In the 1970s it was shown that the intrinsic magnetic relaxation in metals is different from that in magnetic insulators. The Gilbert damping coefficient $G$ in metals is caused by incoherent scattering of electronhole pair excitations by phonons and magnons. The electron-hole pair excitations are either accompanied by a spin flip or the spin remains unchanged. The spin-flip excitations are caused by the exchange interaction between the magnons and itinerant electrons $(s-d$ exchange interaction [2]). The nonspin-flip excitations are caused by the spin-orbit interaction which leads to a dynamic redistribution of electrons in the electron $k$-momentum space [3].

Magnetic multilayers provide a special case where the dynamic interaction between the itinerant electrons and the magnetic moments in ultrathin films offers new exciting possibilities. This is currently one of the main topics of the basic research of magnetic nanostructures and shows much promise for spintronics applications. It has been shown in a number of recent experiments, using either pillar shape nanoscopic samples [4] or point contact geometries [5], that the magnetization reversal can be driven by a spinpolarized current flowing perpendicular to the magnetic layers. Slonczewski [6] showed that a transfer of vectorial spin accompanying an electric current flowing through the interfaces of two magnetic films separated by a nonmagnetic metallic spacer (magnetic double layer) can result in "negative Landau-Lifshitz-like" relaxation torques. This leads, for sufficiently high current densities, to spontaneous magnetization precession and switching phenomena [7]. Berger [8] evaluated the role of the $s-d$ exchange interaction in systems consisting of two magnetic layers separated by a nonmagnetic spacer using a somewhat different approach. One magnetic layer was assumed to be static, and the direction of its magnetic moment determined the axis of the static equilibrium. Magnons were introduced by allowing the magnetic moment of the second (thinner) layer to precess around the equilibrium direction. The itinerant electrons entering the thin ferromagnetic layer through a sharp interface cannot immediately accommodate the direction of the precessing magnetization. Berger showed that this leads to an additional exchange torque which is directed towards the equilibrium axis, and represents an additional Gilbert relaxation term. This relaxation torque is confined to the vicinity of the nonmagnetic/magnetic layer interface. The additional Gilbert relaxation term was calculated using the conservation of the total angular momentum; this means that the electrons in the nonmagnetic spacer have to flip from up to down as a magnon is annihilated in the thin magnetic layer, and vice versa. The relaxation equations for spin-up and spin-down electrons in the spacer layer were obtained using Fermi's golden rule, which has to include the change in energy of an electron when emitting or absorbing a magnon, and the spin-up and spin-down Fermi level shifts.

The resulting relaxation torque in a magnetic double layer structure contributes to an additional ferromagnetic 
resonance (FMR) linewidth [8], $\Delta H_{\text {add }}$, which is proportional to

$$
\Delta H_{\mathrm{add}} \sim(\Delta \mu+\hbar \omega),
$$

where $\Delta \mu=\Delta \mu_{\uparrow}-\Delta \mu_{\downarrow}$ is the difference in the spin-up and spin-down Fermi level shifts, and $\omega$ is the microwave angular frequency. The sign of $\Delta \mu$ depends on the direction of the dc current passing through the film interfaces. This term involves the spin transfer by conduction processes resulting in a net exchange relaxation torque which depends on the density of the perpendicular current and upon the spin-up and spin-down mean-free paths. This part corresponds to Slonczewski's prediction of the currentinduced precession. The second term in Eq. (2) is not present in Slonczewski's model. This term can be obtained by using the full dynamic treatment of the $s$ - $d$ exchange interaction, where magnons are included explicitly in the relaxation equations for occupation numbers. The second part of Eq. (2) is always positive and proportional to the microwave frequency and does not require a dc current crossing the interfaces. It represents an interface Gilbert damping. In ultrathin magnetic films the interface torque is shared by all atomic layers [1]. It follows that the additional FMR linewidth should have an inverse dependence on the film thickness.

Note that both the interface Gilbert damping and the current-driven response $\Delta \mu$ are the results of the same relaxation process [see Eq. (2)]. This means that the measurement of one contribution provides information about the strength of the other. The interface exchange relaxation torque in magnetic multilayers has not, so far, been observed experimentally. The experimental verification of the interface Gilbert damping as proposed by Berger has important implications for spin dynamics in magnetic multilayers. It offers direct access to experimental studies of relaxation torques caused by the transfer of electron angular momentum between the magnetic layers in the presence of the electron-electron exchange interaction. It also has a practical implication. It would allow one to test the suitability of magnetic unpatterned multilayers for their use in systems employing mesoscopic magnetization precession and switching processes, e.g., the spin-wave amplification by stimulated emission of radiation (SWASER) device as proposed by Berger [8]. The purpose of this paper is to investigate the relaxation mechanism associated with magnetic multilayers and to compare the experimental results with predictions obtained from Berger's theoretical model.

Experimental results and discussion. - The preparation of the $\mathrm{Fe} / \mathrm{Au}(001)$ ultrathin film structures was carried out by means of molecular beam epitaxy (MBE). Semiinsulating epiready $\mathrm{GaAs}(001)$ wafers were used as templates for the growth of the metallic structures. The GaAs substrates were prepared by annealing and sputtering cycles and monitored by means of reflection high energy electron diffraction $($ RHEED) until a well-ordered $(4 \times 6)$ reconstruction appeared [9].

Iron films were deposited at room temperature (RT) from a thermal source at a base pressure less than $2 \times$ $10^{-10}$ Torr at a deposition rate of $\sim 1$ Monolayer (ML)/ min. The film thickness was monitored by means of a quartz crystal microbalance and RHEED intensity oscillations. During the growth, approximately 0.6 ML of As segregates on top of the Fe film [9] as identified by $\mathrm{x}$-ray photoemission spectroscopy. When additional metallic layers were deposited, the same amount of As was observed on the surface. The floating As atoms act as a surfactant; no As stays inside the metallic interfaces. The gold was evaporated at RT at a deposition rate of $\sim 1 \mathrm{ML} / \mathrm{min}$. The RHEED oscillations were visible for up to 30 atomic layers. Because of the surfactant As, the RHEED diffraction pattern of $\mathrm{Au}(001)$ showed a $2 \times 2$ reconstruction.

The in-plane FMR (the saturation magnetization and external field are confined to the film plane) experiments were carried out using 10, 24, and $36 \mathrm{GHz}$ systems [10]. The FMR linewidth was measured as the difference in the magnetic fields between the maximum and minimum of the field derivative of the FMR absorption peak, $\partial \chi^{\prime \prime} / \partial H[10]$. The in-plane resonance fields and resonance linewidths were measured as a function of the azimuthal angle $\varphi$ between the external dc magnetic field $\boldsymbol{H}$ and the Fe in-plane cubic axis.

Single Fe ultrathin films with thicknesses of $8,11,16$, 21 , and $31 \mathrm{ML}$ were grown directly on $\mathrm{GaAs}(001)$. They were covered by a 20-ML-thick $\mathrm{Au}(001)$ cap layer for protection in ambient conditions. The FMR measurements were used to determine the in-plane fourfold and uniaxial magnetic anisotropies, $K_{1}$ and $K_{u}$, and the effective demagnetizing field perpendicular to the film surface, $4 \pi M_{\text {eff }}$, as a function of thickness [10]. The results are shown in Fig. 1. The magnetic anisotropies are well described by a linear dependence on $1 / d$, where $d$ is the film thickness. The constant and linear terms represent the bulk and interface magnetic properties, respectively. The ultrathin Fe films grown on GaAs(001) have their magnetic properties nearly equal to those in the bulk Fe, modified only by sharply defined interface anisotropies (see the caption of Fig. 1), indicating that the Fe layers are of a high crystalline quality with well defined interfaces. The in-plane interface uniaxial anisotropy $K_{u}^{s}$ is caused by hybridization of the valence band electrons of Fe with the dangling bonds of GaAs(001) [11].

The line shape of the FMR peaks is Lorentzian and the FMR linewidths are small (under 100 Oe for our microwave frequencies) and only weakly dependent on the film thickness. The reproducible magnetic anisotropies and small FMR linewidths provide an excellent opportunity for investigation of the nonlocal relaxation processes in magnetic multilayer films. The studies were carried out using a simple multilayer structure which consists of only two magnetic layers separated by a 


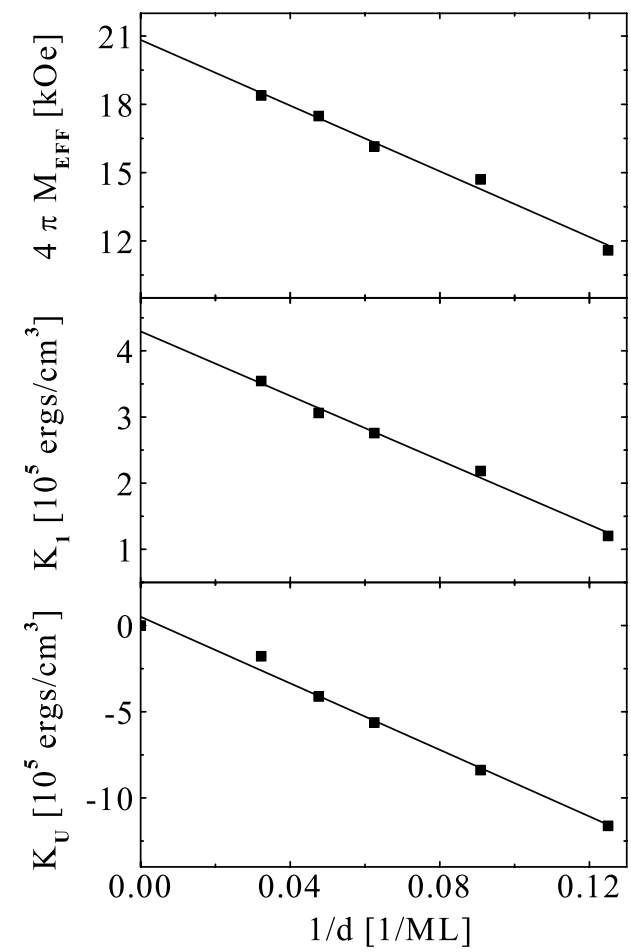

FIG. 1. The effective demagnetizing field perpendicular to the film surface $4 \pi M_{\text {eff }}$ and the in-plane fourfold (cubic) $K_{1}$ and uniaxial $K_{u}$ anisotropies are plotted as a function of $1 / d$ for single $\mathrm{Fe}$ films, where $d$ is the film thickness. The Fe films were grown on $\operatorname{GaAs}(001)$ and covered by $20 \mathrm{ML}$ of $\mathrm{Au}(001) .4 \pi M_{\mathrm{eff}}=4 \pi M_{s}-\frac{2 K_{\perp}^{s}}{M_{s} d}$, where $M_{s}$ is the saturation magnetization and $K_{\perp}^{s}$ is the interface uniaxial perpendicular anisotropy. The fourfold and uniaxial anisotropies are expressed as $K_{1} \cos ^{2}(\varphi) \sin ^{2}(\varphi)$ and $-K_{u} \cos ^{2}\left(\varphi-\varphi_{1 \overline{1}}\right)$, respectively. $\varphi$ is the angle between the external field $\boldsymbol{H}$ and the Fe cubic axis. $\varphi_{1 \overline{1}}$ is the angle of the in-plane hard uniaxial axis. $4 \pi M_{s}=$ $20.8 \mathrm{kOe}, K_{\perp}^{s}=0.9 \mathrm{ergs} / \mathrm{cm}^{2}, K_{1}^{\text {bulk }}=4.3 \times 10^{5} \mathrm{ergs} / \mathrm{cm}^{3}$, $K_{1}^{s}=-0.02 \mathrm{ergs} / \mathrm{cm}^{2}$, and $K_{u}^{s}=-0.07 \mathrm{ergs} / \mathrm{cm}^{2}$, where the superscript $s$ stands for the interface part of the corresponding anisotropies.

nonmagnetic spacer, usually referred to as a magnetic double layer.

The thin Fe films which were studied in the single layer structures were regrown as a part of the double layer structures. The thin Fe film was separated from the second thick Fe layer of 40 ML thickness by a 40 ML thick Au spacer. The double layers were covered by a $20 \mathrm{ML}$ $\mathrm{Au}(001)$ layer for protection under ambient conditions. The complete structure was $\mathrm{GaAs} / \mathrm{Fe}(8,11,16,21,31) /$ $40 \mathrm{Au} / 40 \mathrm{Fe} / 20 \mathrm{Au}(001)$, where the integers represent the number of MLs. The thickness of the Au spacer layer was much smaller than the spin-diffusion length $[12,13]$, and therefore did not affect the spin transport between the magnetic layers.

The interface magnetic anisotropies separated the FMR field of the thin layer from the thick layer by a big margin (see Fig. 2a). That allowed us to carry out the FMR measurements of the thin Fe films in the double layer structures
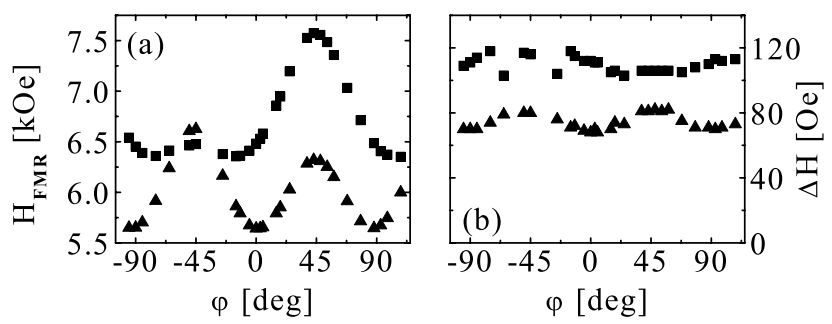

FIG. 2. (a) The FMR field, $H_{\mathrm{FMR}}$, and (b) FMR linewidth, $\Delta H$, as a function of the azimuthal angle $\varphi$ for the GaAs/ $16 \mathrm{Fe} / 40 \mathrm{Au} / 40 \mathrm{Fe} / 20 \mathrm{Au}$ structure at $f=36 \mathrm{GHz}$, where the integers represent the number of ML. The in-plane hard uniaxial axis corresponds to $\varphi=45^{\circ}$. The squares ( $\left.\boldsymbol{\square}\right)$ correspond to the $16 \mathrm{ML}$ Fe film and the triangles $(\boldsymbol{\Delta})$ correspond to the $40 \mathrm{ML}$ Fe film.

with the thick Fe film possessing a negligible angle of precession. The FMR linewidths in single and double layer structures were only weakly dependent on the azimuthal angle $\varphi$ of the saturation magnetization with respect to the in-plane crystallographic axes (see Fig. 2b). The thin Fe film in the single and double layer structures had the same FMR field (see Fig. 3a), showing that the interlayer exchange coupling [1] through the 40-ML-thick Au spacer was negligible, and the magnetic properties of the Fe films grown by $\mathrm{MBE}$ on well-prepared GaAs substrates were fully reproducible.

The FMR linewidth in the thin films always increased in the presence of a thick layer (see Fig. 3b). The additional FMR linewidth, $\Delta H_{\text {add }}$, followed an inverse dependence on the thin film thickness $d$ (see Fig. 4a). The $1 / d$ dependence of $\Delta H_{\text {add }}$ shows that the nonlocal contribution to the FMR linewidth originates at the film interface. $\Delta H_{\text {add }}$ deviates from the straight line mainly for the thinnest $\mathrm{Fe}$ layer, $d=8 \mathrm{ML}$. This can be expected. According to Berger [8], the $1 / d$ dependence fails when the film thickness is comparable to the length given by $\pi /\left(k_{\uparrow}-k_{\downarrow}\right)$, where $k_{\uparrow \downarrow}$ are the spin-up and spin-down Fermi surface wave numbers. A gradual deviation of $\Delta H_{\text {add }}$ from the $1 / d$ dependence indicates that the range of our film thicknesses approaches that limit.
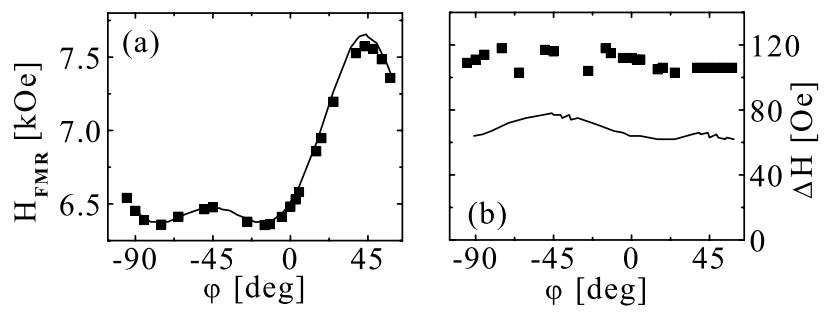

FIG. 3. (a) The FMR field, $H_{\mathrm{FMR}}$, and (b) FMR linewidth, $\Delta H$, for the $16 \mathrm{ML}$ Fe film as a function of the azimuthal angle $\varphi$ at $f=36 \mathrm{GHz}$. The solid lines correspond to the GaAs/ $16 \mathrm{Fe} / 20 \mathrm{Au}$ structure, and the squares $(\boldsymbol{\square})$ correspond to the $\mathrm{GaAs} / 16 \mathrm{Fe} / 40 \mathrm{Au} / 40 \mathrm{Fe} / 20 \mathrm{Au}$ structure. The integers represent the number of MLs. 

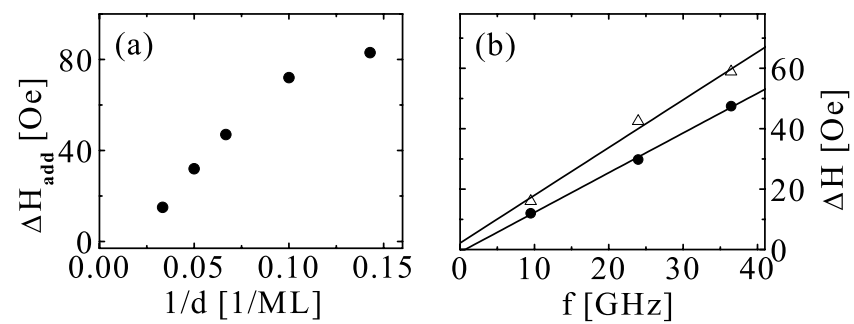

FIG. 4. (a) The dependence of the additional FMR linewidth, $\Delta H_{\text {add }}$, along the $\mathrm{Fe}$ cubic axis on $1 / d$ at $f=36 \mathrm{GHz}$, where $d$ is the thickness of the thin Fe films. (b) The FMR linewidth, $\Delta H$, as a function of the microwave frequency $f$. The triangles $(\triangle)$ correspond to the $16 \mathrm{ML} \mathrm{Fe}$ film in the single layer structure. The dots $(\bigcirc)$ show the additional FMR linewidth, $\Delta H_{\text {add }}$, for the $16 \mathrm{ML}$ Fe film. The solid lines are linear fits to the data.

The frequency dependence of the FMR linewidth of the single Fe film (16 ML) is shown in Fig. 4b. The FMR linewidth is linearly dependent on the microwave angular frequency $\omega=2 \pi f, \Delta H=\Delta H(0)+1.16 \frac{\omega}{\gamma} \frac{G}{\gamma M_{s}}$, where $\Delta H(0)$ is caused by structural imperfections [1]. The corresponding Gilbert damping parameter $G$ and zerofrequency offset $\Delta H(0)$ were found to be $(1.26 \pm 0.12) \times$ $10^{8} \mathrm{~s}^{-1}$ and $2 \pm 4 \mathrm{Oe}$, respectively. Within the accuracy of our measurements (a few Oe) the zero frequency offset $\Delta H(0)$ can be considered to be zero. This means lattice imperfections do not contribute to the FMR linewidth and therefore the measured $G$ is the intrinsic Gilbert damping. This is a unique result not commonly reported for ultrathin films [14].

The linear dependence of $\Delta H_{\text {add }}$ on the microwave frequency with no zero-frequency offset (see Fig. 4b) is a vital result of our studies. It means that the additional contribution to the FMR linewidth can be described by the interface Gilbert damping, $G^{s}$. This is in perfect agreement with Berger's predictions.

The interface Gilbert damping for the 16-ML-thick film was found to be weakly dependent on the crystallographic direction; $G^{s}=(1.03 \pm 0.05) \times 10^{8} \mathrm{~s}^{-1}$ along the cubic axis. Note that its strength is comparable to the intrinsic Gilbert damping in the single Fe film $\left(G=1.26 \times 10^{8} \mathrm{~s}^{-1}\right)$.

In summary, the FMR results on single and double layer structures grown on $\mathrm{GaAs}(001)$ indicate a high crystalline quality of the Fe layers with magnetic anisotropies corresponding to bulk $\mathrm{Fe}$, modified only by interface anisotropies. The reproducible magnetic anisotropies and small FMR linewidths provided an excellent opportunity to investigate nonlocal relaxation processes in ultrathin $\mathrm{Fe}$ films in the presence of a static Fe layer.

The main result of our measurements is that ultrathin Fe films in magnetic double layers acquire an additional interface Gilbert damping term. This is in excellent agree- ment with Berger's prediction of interface nonlocal Gilbert damping in magnetic multilayers. This result has farreaching consequences. Measurements of the nonlocal Gilbert damping offer the possibility to carry out quantitative studies of the relaxation torques caused by transfer of electron angular momentum between magnetic layers in the presence of the electron-electron exchange interaction. It also has practical implications. By using these studies, one should be able to test the suitability of magnetic unpatterned multilayers for potential applications in nanoscopic devices employing mesoscopic magnetization precession and switching processes. It is also worthwhile to point out that the nonlocal Gilbert damping in double $\mathrm{Fe}$ layers grown on GaAs, as shown in this Letter, is a strong effect when compared to the intrinsic Gilbert damping in single Fe layers. Therefore these structures can offer attractive opportunities for microwave applications such as SWASER [8].

The authors thank K. Myrtle, K. Ajdari, and J.F. Cochran for their assistance during the course of this work and L. Berger for valuable discussions. Financial support from the Natural Sciences and Engineering Research Council of Canada (NSERC) and the Canadian Institute for Advanced Research (CIAR) is gratefully acknowledged. B.H. acknowledges the support by the Alexander von Humboldt Foundation.

[1] B. Heinrich and J. F. Cochran, Adv. Phys. 42, 523 (1993).

[2] B. Heinrich, D. Fraitová, and V. Kamberský, Phys. Status Solidi 23, 501 (1967).

[3] V. Kamberský, Can. J. Phys. 48, 2906 (1970).

[4] J. A. Katine, F. J. Albert, R. A. Buhrman, E. B. Myers, and D. C. Ralph, Phys. Rev. Lett. 84, 3149 (2000).

[5] M. Tsoi, A. G. M. Jansen, J. Bass, W.-C. Chiang, M. Seck, V. Tsoi, and P. Wyder, Phys. Rev. Lett. 80, 4281 (1998).

[6] J. C. Slonczewski, J. Magn. Magn. Mater. 159, L1 (1996).

[7] J. C. Slonczewski, J. Magn. Magn. Mater. 195, L261 (1999).

[8] L. Berger, Phys. Rev. B 54, 9353 (1996).

[9] T. L. Monchesky, B. Heinrich, R. Urban, K. Myrtle, M. Klaua, and J. Kirschner, Phys. Rev. B 60, 10242 (1999).

[10] B. Heinrich and J. A. C. Bland, Ultrathin Magnetic Structures II (Springer-Verlag, Berlin, 1994), Sec.: "Radio Frequency Techniques."

[11] J. J. Krebs, B. T. Jonker, and G. A. Prinz, J. Appl. Phys. 61, 2596 (1987).

[12] A. Fert, J.-L. Duvail, and T. Valet, Phys. Rev. B 52, 6513 (1995).

[13] A. Enders, T. Monchesky, K. Myrtle, R. Urban, B. Heinrich, J. Kirschner, X.-G. Zhang, and W. Butler, J. Appl. Phys. 89, 7110 (2001).

[14] Z. Celinski and B. Heinrich, J. Appl. Phys. 70, 5935 (1991). 